\title{
Genetic testing and counselling
}

\author{
Raymond Liang *, MD, FRCP
}

Department of Medicine, Hong Kong Sanatorium and Hospital, Happy Valley, Hong Kong

Hong Kong Med J 2018;24:328-9

*Corresponding author: rliang@hksh.com

DOI: 10.12809/hkmj185081

Genetic testing is an important and rapidly developing field. One third of paediatric medical conditions are related to genetic abnormalities. For adults, most medical conditions are likely the result of the interaction between genetic and environmental factors.

Advances in medical technology mean that genetic testing is now readily available for disease and carrier state diagnosis, risk assessment, prognostic determination, and treatment response prediction for various different diseases. ${ }^{1}$ Available tests include single-gene tests, gene panel testing, and whole genome sequencing. The most appropriate test for a particular clinical situation depends very much on the disease and the test indications. The obtained test results may provide useful information for guiding patient management and for assisting counselling of the patient and their family.

The usefulness of a genetic test in predicting future development of a disease depends on many variables, including the penetrance and expressivity of the clinical phenotype, which may be different between high-risk and low-risk populations. Largescale or long-term studies are required, not only to accurately identify patients who are at high risk of developing a particular disease, but also to provide these patients with appropriate treatment and counselling. This is highlighted in a recent 10 -year study on cardiac genetics in Hong Kong Chinese patients. $^{2}$ For patients identified as high risk, a long-term management plan must be formulated, including aggressive disease screening initiated at an earlier age and advice on lifestyle modifications. Surgical or medical intervention may also be considered. At the same time, related psychosocial problems must also be managed.

For couples with an increased risk of a particular genetic condition, pre-implantation genetic testing can be done on embryos obtained from in vitro fertilisation. ${ }^{3}$ This permits selection of unaffected embryos for implantation. The technique is applicable to hereditary diseases, such as thalassaemia and haemophilia, as well as hereditary cancer syndromes, including breast and colon cancers. ${ }^{4}$ This is usually followed by confirmation by prenatal genetic testing after pregnancy.

Prenatal genetic testing is done to determine whether the fetus is affected by the specific parental genetic abnormality. Specimens are conventionally obtained by chorionic villus sampling or amniocentesis. These procedures are invasive and are not without risk to the mother and the baby, as they are associated with a very small but significant risk of abortion. Prenatal genetic testing is used routinely for parents who are carriers of thalassaemia or haemophilia, and is also used as a complement to pre-implantation genetic testing for many other genetic diseases. Prenatal genetic screening can now be done by analysing fetal cell-free DNA in maternal blood. The technique has been used to detect chromosomal abnormalities such as trisomy 21 and other genetic abnormalities. ${ }^{5}$ However, it is recommended that positive results must always be confirmed by amniocentesis.

Genetic testing must not be done lightly. It must always be coordinated with appropriate genetic counselling by trained personnel, so that the patient and the family can comprehend the meanings and implications of a positive or negative test result. Because genetic testing is often associated with many ethical and psychosocial issues, professional genetic counselling is important. Test results may have important consequences to the family, including genetic discrimination. There are also ethical issues surrounding genetic testing of children. In the United States, legal protection has already been implemented to prevent health insurance providers and employers from using genetic information. However, in many other jurisdictions, including Hong Kong, such protection is not yet available.

Genetic techniques have recently been applied to the therapy of hereditary and acquired diseases. We are witnessing signs of success with gene therapy for haemophilia. ${ }^{6}$ Chimeric antigenic receptor $\mathrm{T}$ cell therapy works by manipulating genetically the $\mathrm{T}$ cells of patients and this mode of treatment has proven useful in the treatment of acute B lymphoblastic leukaemia.?

Genetic technology has opened up a new horizon for the diagnosis and management of hereditary and acquired human diseases. Rapid development in this area is anticipated in the years to come.

\section{Declaration}

The author has disclosed no conflicts of interest.

\section{References}

1. Chan KC, Woo JK, King A, et al. Analysis of plasma Epstein- 
Barr virus DNA to screen for nasopharyngeal cancer. N Engl J Med 2017;377:513-22.

2. Mak CM, Chen SP, Mok NS, et al. Genetic basis of channelopathies and cardiomyopathies in Hong Kong Chinese patients: a 10-year regional laboratory experience. Hong Kong Med J 2018;24:340-9.

3. Bodurtha J, Strauss JF 3rd. Genomics and perinatal care. N Engl J Med 2012;366:64-73.

4. Lee VC, Chow JF, Lau EY, et al. Preimplantation genetic diagnosis for hereditary cancer syndrome: local experience. Hong Kong Med J 2016;22:289-93.
5. Sun K, Jiang P, Chan $\mathrm{KC}$, et al. Plasma DNA tissue mapping by genome-wide methylation sequencing for noninvasive prenatal, cancer and transplantation assessment. Proc Natl Acad Sci U S A 2015;112:e550312.

6. Rangarajin S, Walsh L, Lester W, et al. AAV5-factor VIII gene transfer in severe hemophilia A. N Engl J Med 2017;377:2519-30.

7. Park JH, Rivière I, Gonen $\mathrm{M}$, et al. Long-term follow-up of CD19 CAR therapy in acute lymphoblastic leukemia. N Engl J Med 2018;378:449-59. 